\title{
PERFORMANCE OF THE ATRAKTATM MOSQUITO LURE IN COMBINATION WITH DYNATRAP $®$ (MODELS DT160 AND DT700) AND A CDC TRAP (MODEL 512)
}

\author{
AMY JUNNILA', MOHAMED M. TRAORE', KAREN MACKENZIE², \\ JEROME A. HOGSETTE ${ }^{3}$, DANIEL L. KLINE ${ }^{3}$, ABOUBAKR S. KONE', \\ RABIATOU A. DIARRA', GERGELY PETRANYI', ISSA TROARE', PENDA \\ SANGARE', ASSITAN DIAKITE', SEKOU F. TRAORE', VASILIY D. \\ KRAVCHENKO ${ }^{1,4}$, RUII-DE XUE $^{5}$, EDITA E. REVAY $^{1}$ \\ AND GUNTER C. MULLER' \\ 'Malaria Research and Training Centre, Faculty of Medicine, Pharmacy and \\ Odonto-Stomatology, University of Sciences, Techniques and Technology of \\ Bamako, BP 1805 Bamako, Mali
}

\begin{abstract}
${ }^{2}$ Woodstream Corp., 2226 Sarno Rd Ste 104, Melbourne FL, 32935, USA
${ }^{3}$ USDA-ARS, Center for Medical, Agricultural and Veterinary Entomology, 1600 S.W. 23 ${ }^{\text {rd }}$ Drive, Gainesville, FL, 32608, USA

${ }^{4}$ The Steinhardt Museum of Natural History, Israel National Center for Biodiversity Studies and Department of Zoology Tel Aviv University, Tel Aviv, 6997801, Israel
\end{abstract}

${ }^{5}$ Anastasia Mosquito Control District, 120 EOC, St. Augustine, FL 32092, USA

Subiect Editor: Yong-Xing Jiang

\begin{abstract}
The performance of the three-part mosquito lure ATRAKTA (1-octen-3-ol, ammonium bicarbonate, and lactic acid) was evaluated in two DynaTrap commercial mosquito traps (models DT160 and DT700) as well as in one model of CDC trap (model 512). Lures were evaluated fresh from the factory, after being aged in functioning traps under field conditions, and after prolonged storage in the packaging (aged for 30 days aged in functioning traps before being tested in the DynaTrap models; and two years stored in the packaging before being tested in CDC traps). The primary study questions were whether the addition of lures would increase efficacy of various trap types and whether lures would retain effectiveness after a lengthy stay on the shelf or in traps. To do this, traps with no lures, new lures and old lures were used to trap three mosquito species (Aedes albopictus, Culex quinquefasciatus, and Anopheles gambiae) in the field in West Africa Mali, the first two species are also common North American nuisance mosquitoes. The addition of ATRAKTA lures aged 30 days to both DynaTrap ${ }^{\circledR}$ models, and ATRAKTA lures aged two years in the packaging to the CDC trap significantly increased catches of female Cx. quinquefasciatus and Ae. albopictus mosquitoes. Aged lures did not significantly lose their attraction in comparison to lures fresh from the factory. The addition of lures to traps resulted in slight increases in catches of An. gambiae, but these were not statistically significant. No effect of any lures on males was observed.
\end{abstract}

Key Words: Atrakta, attractant, Dynatrap, CDC trap, Anopheles gambiae, Aedes, albopictus, Culex quinquefasciatus

\section{INTRODUCTION}

The success of Integrated Vector Management (IVM) programs is generally measured using surveillance traps which can be costly. In a previous study, (Traore et al., 2021), we demonstrated that the DynaTrap (Model DT160) did just as well or better at trapping Aedes albopictus, Culex quinquefasciatus, and Anopheles gambiae s.l. as more 
costly CDC trap models. We hypothesized that the success of these surveillance traps at catching female mosquitoes could be significantly increased with a good lure. Common attractants in mosquito surveillance traps include light, host-mimicking $\mathrm{CO}_{2}$, and an array of volatile compounds that emanate from plants or fungi, such as octenol (Kline, 1994), or from human skin, such as L-lactic Acid and Ammonia (Acree et al., 1968; Kline et al., 1990; Geier et al., 1999; Hoel et al., 2007). The Atrakta pod lure (Woodstream Corp., Lititz, PA, USA) is a combination of octenol (1-octen-3-ol), L-lactic acid and ammonia. All three of these compounds have been identified as mosquito attractants separately and/or in various combinations, to varying degrees of attractiveness, depending on combinations as well as mosquito species. The goal of the triple combination is to have a broader range of attraction than the individual compounds alone.

The effect of the ATRAKTA pod lures in traps was evaluated against $C x$. quinquefasciatus, Ae. albopictus and An. gambiae. These first two are nuisance mosquito species in North America but are also important disease vectors there and in other countries, as well (Bhattacharya et al. 2016; Gratz 2004). They are essentially cosmopolitan in their distribution (Farajollahi et al., 2011; Kraemer et al., 2015). An. gambiae s.l. is an important malaria vector in Africa (Rosenthal et al. 2019). The main questions were how much the addition of the 3-part ATRAKTA pod lure would increase trap catches of these three species and if a fresh and a 30day old lure would perform equally well in a DynaTrap model DT160 or DT700. Model 512 CDC traps equipped with either a fresh or a 2-year-old package-aged lure served as a standard trap.

\section{MATERIALS AND METHODS}

Study sites. Trials with Cx. quinquefasciatus were conducted in suburban Bamako $\left(-7.89551508800^{\circ} \mathrm{N} 12.65701558800^{\circ} \mathrm{W}\right)$ on a quiet residential road bordered on both sides with drainage ditches. The traps were set up in a row, along 1 of the ditches, sus- pended $1.5 \mathrm{~m}$ above the ground from tripods, positioned between the ditch and the fences/walls of the nearby properties. The traps were 1 to $2 \mathrm{~m}$ from the ditch and 25 $m$ apart.

Trials with Ae. albopictus were conducted in downtown Bamako $\left(-7.92503622500^{\circ} \mathrm{N}\right.$ $\left.12.65316964500^{\circ} \mathrm{W}\right)$ on public parkland along the River Niger. The traps were set up in a row, along the river, suspended $1.5 \mathrm{~m}$ above the ground from tripods, positioned between herbaceous plants shaded by large trees. The traps were 20 to $30 \mathrm{~m}$ away from the river and $25 \mathrm{~m}$ apart.

Trials with An. gambiae s.l. were conducted in Kenieroba $\left(-8.32928630400^{\circ} \mathrm{N}\right.$ $12.11465570600^{\circ}$ W) $60 \mathrm{~km} \mathrm{SW}$ of Bamako on the flood plain of the River Niger in naturally irrigated rice fields. The traps were set up on tripods $1.5 \mathrm{~m}$ above the ground parallel to an irrigation ditch in which An. gambiae s.l. were breeding. Traps were placed $25 \mathrm{~m}$ apart.

\section{Traps and lures}

The following traps were used in the study: DynaTrap model DT160, light source: Cold Cathode Fluorescent Light UV (Woodstream Corp., Melbourne FL, USA); DynaTrap model DT700, light source: LED UV (Woodstream Corp., Melbourne FL, USA); CDC Trap model 512, light source, incandescent light bulb (John W. Hock, Gainesville FL, USA). ATRAKTA pod mosquito lures (Lactic Acid - 63.69\%, 1-octen-3-ol $73.36 \%$, Ammonium; Bicarbonate - 100\%; Woodstream Corp., Melbourne FL, USA; Fig. 1), both fresh from the factory and aged for 30 days in the field (12 hr per night) in functioning model DT160 DynaTrap traps before testing began, were used in the two DynaTrap models; ATRAKTA pod mosquito lures both fresh from the factory and aged two years in storage (off the shelf) were used in the model 512 CDC trap.

Trial Design. In total, nine trials, two at the same time for Culex quinquefasciatus and for Aedes albopictus but in two different habitats, and the one for Anopheles gambiae, were conducted during 2020. Trial I with DynaTrap model DT160 was carried out in early to 


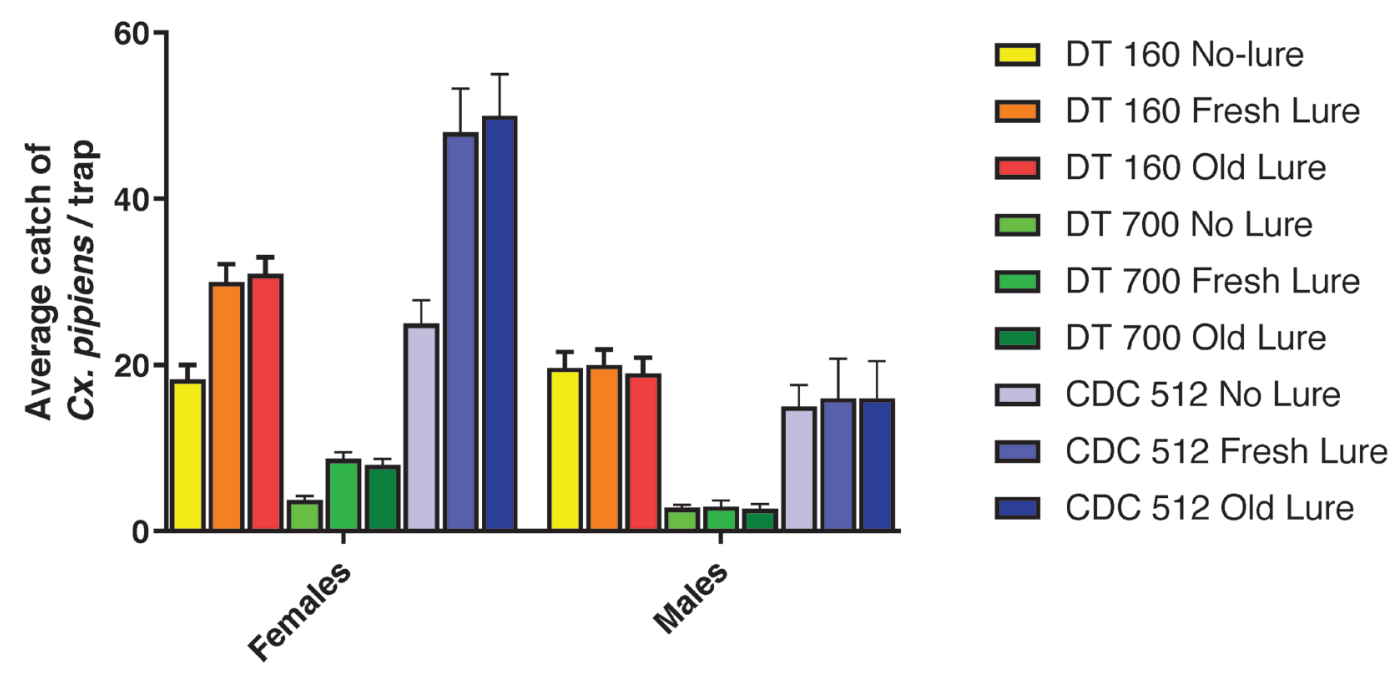

Fig. 1. Effect of the ATRAKTA pod lure on DynaTrap DT160, DT700 and CDC model 512 traps.

mid February over 14 consecutive days and nights. Trial II with DynaTrap model DT700 was carried out during mid to end of February over 10 consecutive days and nights. Trial III with CDC trap model 512, was carried out during early to mid June over 10 consecutive days and nights. Trials with An. gambiae s.l. were carried out in mid to late October; Trial I with DynaTrap model DT160 lasted 14 consecutive nights, Trial II with DynaTrap model DT700 lasted 10 consecutive nights and Trial III with CDC trap model 512 lasted 10 consecutive nights.

During each trial, six traps of the same kind were operated simultaneously. Two traps each were operated without lures (controls), two traps with fresh lures and two traps with old lures (either aged 30 days in traps or two years in storage). The differently baited traps were placed on alternate tripods in a row and positions were rotated daily to avoid positional bias. All traps for each experiment were operated over night from 18:00 to 7:00 h. Collection nets were emptied the following morning. The lures were placed in the special lure chambers in the two commercial traps while the lure was placed in the CDC trap within the collection bag $10 \mathrm{~cm}$ below the fan. After each trial, the used lures were discarded.

Statistics. The mean numbers of mosquitoes caught per trap (male and female) per night (24 nights) for each trial were calculated from replicates of the experiments and were analyzed using two-way ANOVA followed by a Sidak post-hoc test to rank significance levels. Differences were said to be significant at $\mathrm{P}<0.05$. Analysis was conducted using GraphPad Prism 8.00 for windows (GraphPad Software, La Jolla California, USA). The mean numbers of mosquitoes $( \pm$ SEM $)$ and P-value of the comparisons are reported in Tables 1 through 3 .

\section{RESULTS}

The mean numbers of Cx. quinquefasciatus females captured by the DynaTrap model DT160 with fresh and 30-day aged ATRAK-

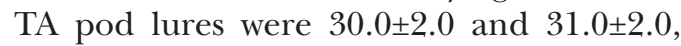
respectively; which were significantly greater than the mean of $18.3 \pm 1.7$ captured by the control trap with no lure (Fig. 1, Table 1). This represented an increase of 62 and $68 \%$, respectively. The mean numbers of $C x$. quinquefasciatus females captured by the DynaTrap model DT700 with fresh and 30-day aged ATRAKTA pod lures were $8.7 \pm 0.8$ and $8.00 \pm 0.7$, respectively, which were significantly greater than the mean of $3.8 \pm 0.5$ captured by the control trap with no lure (Fig. 1 , Table 2). This represented an increase of 131 and $112 \%$, respectively. The mean numbers of $C x$. quinquefasciatus females captured 
Table 1. The effect of ATRAKTA pod lure type (no lure, fresh lure, or old lure) on mean numbers of Cx. quinquefasciatus females and males $( \pm$ SEM) caught per DynaTrap DT160, DynaTrap DT700, and the CDC trap model 512 during the trials on 14, 10, and 10 consecutive nights from early to mid February-late October 2020.

\begin{tabular}{|c|c|c|c|c|c|}
\hline \multirow[b]{2}{*}{ Trap Model } & \multirow[b]{2}{*}{ Sex } & \multirow[b]{2}{*}{ Lure comparisons } & \multirow{2}{*}{$\begin{array}{c}\text { Mean \# } \pm \text { SEM } \\
\text { (Lure } 1)\end{array}$} & \multirow{2}{*}{$\frac{\text { Mean } \# \pm \text { SEM }}{\text { (Lure } 2)}$} & \multirow{2}{*}{$\begin{array}{c}\text { Adjusted } \\
\text { P Value }\end{array}$} \\
\hline & & & & & \\
\hline \multirow[t]{6}{*}{ DT160 } & \multirow[t]{3}{*}{ Females } & No-lure vs. Fresh Lure & $18.3 \pm 1.7$ & $30.0 \pm 2.0$ & 0.0001 \\
\hline & & No-lure vs. Old Lure & $18.3 \pm 1.7$ & $31.0 \pm 2.0$ & $<0.0001$ \\
\hline & & Fresh Lure vs. Old Lure & $30.0 \pm 2.0$ & $31.0 \pm 2.0$ & 0.9771 \\
\hline & \multirow[t]{3}{*}{ Males } & No-lure vs. Fresh Lure & $19.7 \pm 1.9$ & $20.0 \pm 2.0$ & 0.9993 \\
\hline & & No-lure vs. Old Lure & $19.7 \pm 1.9$ & $19.0 \pm 2.0$ & 0.9919 \\
\hline & & Fresh Lure vs. Old Lure & $20.0 \pm 2.0$ & $19.0 \pm 2.0$ & 0.9771 \\
\hline \multirow[t]{6}{*}{ DT700 } & \multirow[t]{3}{*}{ Females } & No-lure vs. Fresh Lure & $3.8 \pm 0.5$ & $8.7 \pm 0.8$ & $<0.0001$ \\
\hline & & No-lure vs. Old Lure & $3.8 \pm 0.5$ & $8.0 \pm 0.7$ & $<0.0001$ \\
\hline & & Fresh Lure vs. Old Lure & $8.7 \pm 0.8$ & $8.0 \pm 0.7$ & 0.8216 \\
\hline & \multirow[t]{3}{*}{ Males } & No-lure vs. Fresh Lure & $2.8 \pm 0.4$ & $3.0 \pm 0.7$ & 0.9945 \\
\hline & & No-lure vs. Old Lure & $2.8 \pm 0.4$ & $2.7 \pm 0.6$ & 0.9993 \\
\hline & & Fresh Lure vs. Old Lure & $3.0 \pm 0.7$ & $2.7 \pm 0.6$ & 0.9821 \\
\hline \multirow[t]{6}{*}{ CDC-Candescent } & \multirow[t]{3}{*}{ Females } & No-lure vs. Fresh Lure & $25.0 \pm 2.8$ & $48.0 \pm 5.0$ & 0.0007 \\
\hline & & No-lure vs. Old Lure & $25.0 \pm 2.8$ & $50.0 \pm 5.1$ & 0.0002 \\
\hline & & Fresh Lure vs. Old Lure & $48.0 \pm 5.0$ & $50.0 \pm 5.1$ & 0.9830 \\
\hline & \multirow[t]{3}{*}{ Males } & No-lure vs. Fresh Lure & $15.0 \pm 2.6$ & $16.0 \pm 5.0$ & 0.9978 \\
\hline & & No-lure vs. Old Lure & $15.0 \pm 2.6$ & $16.0 \pm 5.0$ & 0.9978 \\
\hline & & Fresh Lure vs. Old Lure & $16.0 \pm 5.0$ & $16.0 \pm 5.0$ & $>0.9999$ \\
\hline
\end{tabular}

Fresh Lure - direct from the package

Old Lure - 30 days old (in DynaTraps); 2-years old (in CDC Traps)

by the CDC model 512 with fresh and 2-yr aged ATRAKTA pod lures were 48.0 45.0 and $50.0 \pm 5.1$, respectively, which were significantly greater than the mean of $25.0 \pm 2.8$ captured by the control trap with no lure (Fig. 1, Table 3). This represented an increase of 94 and $100 \%$, respectively. Ranked trap efficacy in decreasing order for $C x$. quinquefasciatus females was CDC model $512>$ DynaTrap model DT160 > DynaTrap model DT 700.

For each trap type, there was no significant difference between catches of $C x$. quinquefasciatus females when using fresh or any aged ATRAKTA pod lures (Tables 1-3). Catches of Cx. quinquefasciatus males were not significantly affected by using the lures in combination with any trap.

The mean numbers of Ae. albopictus females captured by the DynaTrap model DT160 with fresh and 30-day aged ATRAKTA pod lures were $15.8 \pm 1.4$ and $16.5 \pm 1.3$, respectively, which were significantly greater than the mean of $7.0 \pm 0.7$ captured by the control trap with no lure (Fig. 2, Table 2). This represented an increase of 126.9 and
$137.6 \%$, respectively. The mean numbers of Ae. albopictus females captured by the DynaTrap model DT700 with fresh and 30day aged ATRAKTA pod lures were $4.8 \pm 0.5$ and $4.6 \pm 0.4$, respectively, which were significantly greater than the mean of $1.6 \pm 0.2$ captured by the control trap with no lure (Fig. 2 , Table 2). This represented an increase of 200.0 and $184.4 \%$, respectively. The mean numbers of Ae. albopictus females captured by the CDC model 512 with fresh and 2-yr aged ATRAKTA pod lures were 2.6 \pm 0.3 and $2.9 \pm 0.4$, respectively, which were significantly greater than the mean of $1.4 \pm 0.4$ captured by the control trap with no lure (Fig. 2 , Table 6). This represented an increase of 82.1 and $103.5 \%$, respectively. Ranked trap efficacy in decreasing order for Ae. albopictus females was DynaTrap model DT160 > DynaTrap model DT700 > CDC model 512.

For each trap type, there was no significant difference between catches of Ae. albopictus females when using fresh or any aged ATRAKTA pod lures (Table 2). Catches of Ae. albopictus males were not significantly 
Table 2. The effect of ATRAKTA pod lure type on mean numbers of Ae. albopictus females and males ( \pm SEM) caught per DynaTrap DT160, DynaTrap DT700, and the CDC trap model 512 during the trials on 14, 10, and 10 consecutive nights from early to mid February-late October 2020.

\begin{tabular}{|c|c|c|c|c|c|}
\hline & & & Mean \# \pm SEM & Mean \# \pm SEM & \\
\hline Trap Model & Sex & Lure comparisons & (Lure 1) & (Lure 2) & $\mathrm{P}$ Value \\
\hline \multirow{6}{*}{ DT160 } & \multirow{3}{*}{ Females } & No-lure vs. Fresh Lure & $7.0 \pm 0.7$ & $15.8 \pm 1.4$ & $<0.0001$ \\
\hline & & No-lure vs. Old Lure & $7.0 \pm 0.7$ & $16.5 \pm 1.3$ & $<0.0001$ \\
\hline & & Fresh Lure vs. Old Lure & $15.8 \pm 1.4$ & $16.5 \pm 1.3$ & 0.9452 \\
\hline & \multirow[t]{3}{*}{ Males } & No-lure vs. Fresh Lure & $5.1 \pm 0.6$ & $4.9 \pm 1.2$ & 0.9995 \\
\hline & & No-lure vs. Old Lure & $5.1 \pm 0.6$ & $5.4 \pm 1.1$ & 0.9941 \\
\hline & & Fresh Lure vs. Old Lure & $4.9 \pm 1.2$ & $5.4 \pm 1.1$ & 0.9864 \\
\hline \multirow[t]{6}{*}{ DT700 } & \multirow[t]{3}{*}{ Females } & No-lure vs. Fresh Lure & $1.6 \pm 0.2$ & $4.8 \pm 0.5$ & $<0.0001$ \\
\hline & & No-lure vs. Old Lure & $1.6 \pm 0.2$ & $4.6 \pm 0.4$ & $<0.0001$ \\
\hline & & Fresh Lure vs. Old Lure & $4.8 \pm 0.5$ & $4.6 \pm 0.4$ & 0.949 \\
\hline & \multirow[t]{3}{*}{ Males } & No-lure vs. Fresh Lure & $1.1 \pm 0.2$ & $1.4 \pm 0.4$ & 0.9136 \\
\hline & & No-lure vs. Old Lure & $1.1 \pm 0.4$ & $1.2 \pm 0.4$ & 0.9992 \\
\hline & & Fresh Lure vs. Old Lure & $1.4 \pm 0.4$ & $1.2 \pm 0.4$ & 0.9728 \\
\hline \multirow[t]{6}{*}{ CDC-Candescent } & \multirow[t]{3}{*}{ Females } & No-lure vs. Fresh Lure & $1.4 \pm 0.2$ & $2.6 \pm 0.3$ & 0.0099 \\
\hline & & No-lure vs. Old Lure & $1.4 \pm 0.2$ & $2.9 \pm 0.4$ & 0.0007 \\
\hline & & Fresh Lure vs. Old Lure & $2.6 \pm 0.3$ & $2.9 \pm 0.4$ & 0.8520 \\
\hline & \multirow[t]{3}{*}{ Males } & No-lure vs. Fresh Lure & $0.5 \pm 0.2$ & $0.7 \pm 0.2$ & 0.8869 \\
\hline & & No-lure vs. Old Lure & $0.5 \pm 0.2$ & $0.7 \pm 0.3$ & 0.9377 \\
\hline & & Fresh Lure vs. Old Lure & $0.7 \pm 0.2$ & $0.7 \pm 0.3$ & 0.9991 \\
\hline
\end{tabular}

Fresh Lure - direct from the package

Old Lure - 30 days old (in DynaTraps); 2-years old (in CDC Traps)

affected by using the lures in combination with any trap.

The mean numbers of An. gambiae s.l. females captured by the DynaTrap model DT160 with fresh and 30-day aged ATRAKTA pod lures were $31.4 \pm 2.4$ and $30.0 \pm 2.4$, respectively, which were not significantly greater than the mean of $29.4 \pm 2.3$ captured by the control trap with no lure (Fig. 3, Table 3). This represented an increase of 6.5 and $1.9 \%$, respectively. The mean numbers of An. gambiae s.l. females captured by the DynaTrap model DT700 with fresh and 30-day aged ATRAKTA pod lures were $4.5 \pm 0.5$ and $4.3 \pm 0.5$, respectively, which were not significantly greater than the mean of $4.0 \pm 0.4$ captured by the control trap with no lure (Fig. 3 , Table 3). This represented an increase of 13.0 and $6.3 \%$, respectively. The mean numbers of An. gambiae s.l. females captured by the CDC model 512 with fresh and 2-yr aged ATRAKTA pod lures were $6.0 \pm 0.7$ and $6.0 \pm 0.8$, respectively, which were not significantly greater than the mean of $4.9 \pm 0.5$ captured by the control trap with no lure (Fig.
3, Table 3). This represented an increase of 22.5 and $21.4 \%$, respectively. Ranked trap efficacy in decreasing order for An. gambiae s.l. females was DynaTrap model DT160 > DynaTrap model DT700 > CDC model 512.

For each trap type, there was no significant difference between catches of An. gambiae s.l. females when using fresh or any aged ATRAKTA pod lures (Table 3). Catches of An. gambiae s.l. males were not significantly affected by using the lures in combination with any trap.

\section{DISCUSSION}

Use of an ATRAKTA pod lure in DynaTraps and CDC traps can significantly increase the numbers of female mosquitoes captured, except for An. gambiae.. There was no significant difference between lures that were fresh, and lures aged for 30 days in the traps (or 2 years in the package in the case of CDC traps). The ATRAKTA pod lure significantly increased DynaTrap catches of both nuisance species $C x$. quinquefasciatus and $A e$. albopictus females. 
Table 3. The effect of ATRAKTA pod lure type on mean numbers of An. gambiae females and males ( \pm SEM) caught per Dyna Trap DT160, Dyna Trap DT700, and CDC trap model 512 during the trials on 14, 10, and 10 consecutive nights from early to mid-February-late October, 2020.

\begin{tabular}{|c|c|c|c|c|c|}
\hline \multirow{2}{*}{ Trap Model } & \multirow{2}{*}{ Sex } & \multirow{2}{*}{ Lure comparisons } & \multirow{2}{*}{$\frac{\text { Mean \# } \pm \text { SEM }}{(\text { Lure } 1)}$} & \multirow{2}{*}{$\begin{array}{c}\text { Mean \# } \pm \text { Sem } \\
\text { (Lure 2) }\end{array}$} & \multirow{2}{*}{$\begin{array}{c}\text { Adjusted } \\
\text { P Value }\end{array}$} \\
\hline & & & & & \\
\hline \multirow[t]{6}{*}{ DT160 } & \multirow[t]{3}{*}{ Females } & No-lure vs. Fresh lure & $29.4 \pm 2.3$ & $31.4 \pm 2.4$ & 0.9109 \\
\hline & & No-lure vs. Old lure & $29.4 \pm 2.3$ & $30.0 \pm 2.4$ & 0.9973 \\
\hline & & Fresh-lure vs. Old lure & $31.4 \pm 2.4$ & $30.0 \pm 2.4$ & 0.9727 \\
\hline & \multirow[t]{3}{*}{ Males } & No-lure vs. Fresh lure & $11.4 \pm 2.2$ & $11.90 \pm 2.3$ & 0.9986 \\
\hline & & No-lure vs. Old lure & $11.4 \pm 2.2$ & $11.9 \pm 2.4$ & 0.9982 \\
\hline & & Fresh-lure vs. Old lure & $11.9 \pm 2.3$ & $11.9 \pm 2.4$ & $>0.9999$ \\
\hline \multirow[t]{6}{*}{ DT700 } & \multirow[t]{3}{*}{ Females } & No-lure vs. Fresh lure & $4.0 \pm 0.4$ & $4.5 \pm 0.5$ & 0.8311 \\
\hline & & No-lure vs. Old lure & $4.0 \pm 0.4$ & $4.3 \pm 0.5$ & 0.9740 \\
\hline & & Fresh-lure vs. Old lure & $4.5 \pm 0.5$ & $4.3 \pm 0.5$ & 0.9791 \\
\hline & \multirow[t]{3}{*}{ Males } & No-lure vs. Fresh lure & $2.1 \pm 0.4$ & $2.0 \pm 0.5$ & 0.9982 \\
\hline & & No-lure vs. Old lure & $2.1 \pm 0.4$ & $2.2 \pm 0.6$ & 0.9982 \\
\hline & & Fresh-lure vs. Old lure & $2.0 \pm 0.5$ & $2.2 \pm 0.6$ & 0.9891 \\
\hline \multirow[t]{6}{*}{ CDC-Candescent } & \multirow[t]{3}{*}{ Females } & No-lure vs. Fresh lure & $4.9 \pm 0.5$ & $6.0 \pm 0.7$ & 0.5155 \\
\hline & & No-lure vs. Old lure & $4.9 \pm 0.5$ & $6.0 \pm 0.8$ & 0.5541 \\
\hline & & Fresh-lure vs. Old lure & $6.0 \pm 0.7$ & $6.0 \pm 0.8$ & $>0.9999$ \\
\hline & \multirow[t]{3}{*}{ Males } & No-lure vs. Fresh lure & $1.9 \pm 0.5$ & $2.2 \pm 0.7$ & 0.9890 \\
\hline & & No-lure vs. Old lure & $1.9 \pm 0.5$ & $2.2 \pm 0.7$ & 0.9812 \\
\hline & & Fresh-lure vs. Old lure & $2.2 \pm 0.7$ & $2.2 \pm 0.7$ & $>0.9999$ \\
\hline
\end{tabular}

Fresh Lure - direct from the package

Old Lure - 30 days old (in DynaTraps); 2-years old (in CDC Traps)

The ATRAKTA pod lure did not make statistically significant increases in catches of An. gambiae females. In a 2020 laboratory report by Sierra Research Laboratories, Inc., a similar result was obtained using An. quadrimaculatus where the percentage of recovered mosquitoes (between baited and unbaited traps) numbered only $38 \%$. In France in 2011, trap performance when baited solely with octenol was estimated at only $43 \%$ in trapping An. hyrcanus (Roiz et al., 2012). Essen and colleagues (1994) reported differential attraction of Aedes and Culex mosquitoes to light and octenol baited $\mathrm{CO}^{2}$ traps.

Carbon dioxide is sometimes used as a general attractant (Newhouse et al. 1966) and there are a number of chemical lures on the market that will enhance the attraction of mosquito traps (Bernier et al. 2008). Some of these lures will attract certain mosquito species more than others (Essen et al. 1994; Burkett et al. 2001). The ATRAKTA pod lure was selective in its attraction of $C x$. pipiens quinquefasciatus and Ae. albopictus.
Many mosquito traps, including the ones in the study, use some type of light as an attractant (Kline 1994; Ponlawat et al. 2017). A recent study showed that a new model of DynaTrap, DT 2000, baited with ATRAKTA collected significantly higher numbers of adult mosquitoes and non-targets, compared with the CDC light trap baited with the same lure (Acevedo et al. 2020). It is notable that the DynaTrap DT160 caught significantly more females than the DT700 regardless of lure type. The DynaTrap DT160, utilizes a $3.5 \mathrm{~W} \pm 10 \%$ circular Cold Cathode Fluorescent Light (CCFL) source that produces a wavelength of $365 \mathrm{~nm} \pm 5 \mathrm{~nm}$, drawing $0.4 \mathrm{~A} /$ hour from a $12 \mathrm{~V}$ battery, whereas the DT700 uses UV emanating from 3 small LED bulbs. The better performance of the DynaTrap DT160 could be affected by the differences in the type of UV source, as well as the configuration of the bulb.

In conclusion, ATRAKTA pod 3-part lures can be used to boost trap catches of common nuisance mosquito females, in some cases by well over $100 \%$ either fresh 




Fig. 2. Effect of the ATRAKTA pod lure on DynaTrap DT160, DT700 and CDC model 512 trap catches of Ae. albopictus.

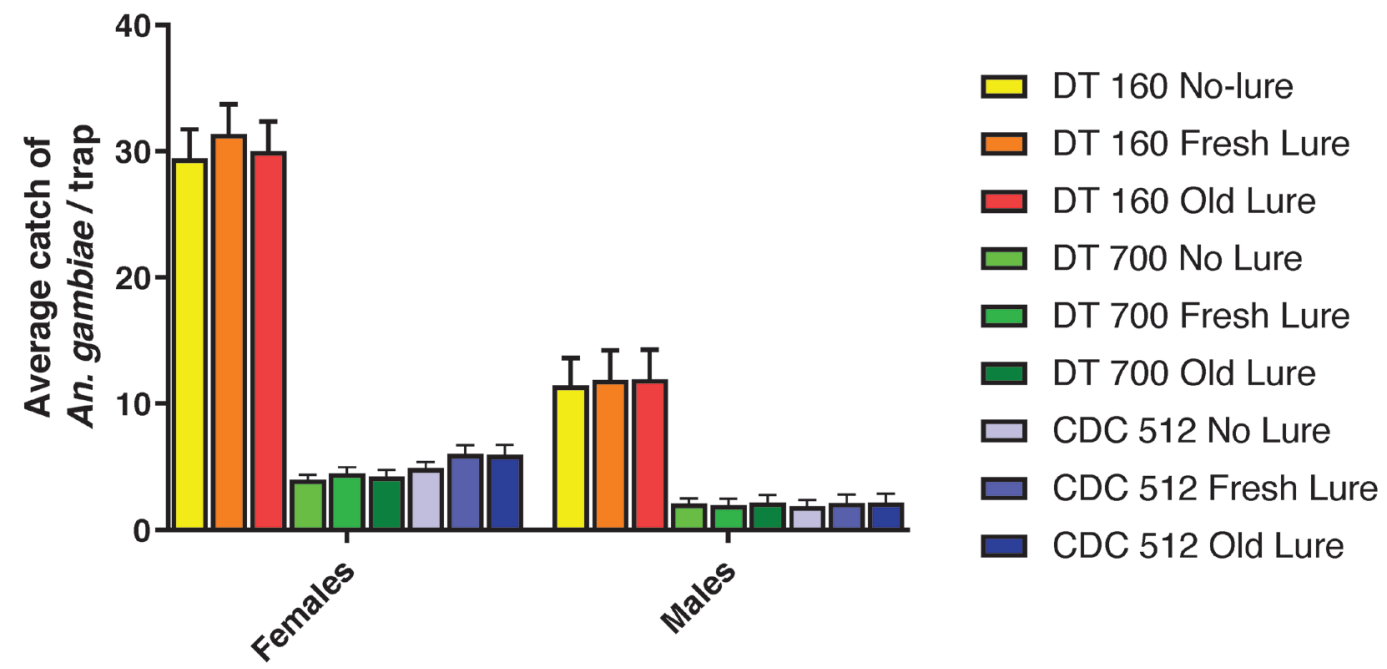

Fig. 3. Effect of the ATRAKTA pod lure on DynaTrap DT160, DT700 and CDC model 512 trap catches of $A n$. gambiaes.

out of the bag, under field conditions, or after prolonged storage periods.

\section{ACKNOWLEDGEMENTS}

The authors wish to thank participating communities in Bamako and Kenieroba, Mali. The trials were in part supported by the ICEMR Vector supplement Grant Number 5U19AI089672.

\section{REFERENCES CITED}

Acevedo N, Efstathion C, Xue RD, Qualls WA. 2020. Comparison of the CDC light trap and the DYNATRAP DT2000 for collection of mosquitoes in semifield and field settings. J Florida M C Assoc. 67:69-72.

Acree F, Turner RB, Gouck HK, Beroza M, Smith N. 1968. L-Lactic acid: a mosquito attractant isolated from humans. Science, 161:1346-1347.

Bhattacharya S, Basu P. 2016. The southern house mosquito, Culex quinquefasciatus: profile of a smart vector. J Entomol Zool Stud, 4(2), 73-81

Bernier UR, Hoel DF, Hogsette Jr JA, Hanafi HA, Kline DL. 2008. Effect of lures and trap placement on 
sand fly and mosquito traps. Proceedings of the Sixth International Conference on Urban Pests. 171-175.

Essen PV, Kemme JA, Ritchie SA, Kay BH. 1994. Differential responses of Aedes and Culex mosquitoes to octenol or light in combination with carbon dioxide in Queensland, Australia. Med Vet Entomol, 8(1), 63-67.

Farajollahi A, Fonseca DM, Kramer LD, Kilpatrick AM. 2011. "Bird biting" mosquitoes and human disease: a review of the role of Culex pipiens complex mosquitoes in epidemiology. Infect, Genet Evol. 11(7), 1577-1585.

Geier M, Bosch OJ, and Boeckh J. 1999. Ammonia as an attractive component of host odour for the yellow fever mosquito, Aedes aegypti. Chem senses 24(6), 647-653.

Gratz, NG. 2004. Critical review of the vector status of Aedes albopictus. Med Vet Entomol 18(3), 215-227.

Hoel DF, Kline DL, Allan SA, Grant A. 2007. Evaluation of Carbon Dioxide, 1-octen-3-ol, and Lactic Acid as baits in Mosquito Magnet M Pro traps for Aedes albopictus in North Central Florida. J Am Mos Cont Assoc 23(1), 11-17.

Kline DL. 1994. Olfactory attractants for mosquito surveillance and control: 1-octen-3-ol. Mosquito News 10(2), 280-287.
Kraemer MU, Sinka ME, Duda KA, Mylne AQ, Shearer FM, Barker CM, Moore CG, Carvalho RG, Coelho GE, Van Bortel W, Hendrickx G. 2015. The global distribution of the arbovirus vectors Aedes aegypti and Ae. albopictus. Elife 4, p.e08347.

Newhouse V, Chamberlain F, Johnson JG, Sudia WD. 1966. Use of dry ice to increase mosquito catches of the CDC-miniature Light Trap. Mosquito News 26(1):30-35.

Roiz D, Roussel M, Muñoz J, Ruiz S, Soriguer R, Figuerola J. 2012. Efficacy of mosquito traps for collecting potential West Nile mosquito vectors in a natural Mediterranean wetland. Am J Tropical Med Hygiene 86(4), 642-648.

Rosenthal PJ, John CC, Rabinovich NR. 2019. Malaria: how are we doing and how can we do better? $A m$ J Trop Med Hyg 100 (2), 239.

Traore MM, Junnila A, Hogsette JA, Kline DL, Mckenzie K, Kravchenko V, Kone AS, Diarra RA, Traore SF, Petranyi G, Sangare P, Diakite A, Troare I, Beier JC, Revay EE, Xue, RD, Muller GC. 2021. Evaluation of Dynatrap DT160 as an inexpensive alternative to CDC traps for adult mosquito monitoring in Mali, West Africa. J Florida Mosq Control Assoc. 68: 38-47. 